CASE 2.-Traumatic tetanus in a negro, aged eighteen years, coming on forty-eight hours after a severe sprain of the muscles of the back. Recovered.

CASE 3.- Traumatic tetanus in a negro, aged fifty years, coming on six days after the foot had been pierced by a crowbar. Recovered.

CASE 4.-Idiopathic tetanus in a negro boy, aged ten years. Recovered

CASE 5.-Tdiopathic tetanus in a Portuguese boy, aged fourteen years, coming on forty-eight hours after plunging into a trough of cold water while heated. Recovered.

The first two and the last one were cases of acute tetanus ; the third was not so severe, but could hardly be classed as chronic tetanus, for at times the spasm was intense; the fourth case was one of chronic tetanus.

The course of treatment which I endeavour to carry out is as follows:-First. The room must be dark and quiet; draughts are to be carefully excluded. Too much stress cannot be placed on this ; the least rush of cold air, flash of light, or even sudden noise, may bring on a spasm. On one occasion the contact of my cold hand when feeling the pulse produced one. Another case of traumatic tetanus was progressing favourably, when suddenly in the night there was an alarm of fire; great noise and confusion ensued, windows were thrown open, and, caused either by that or by the fright, severe spasms came on, in one of which he died in an hour or two.

Second. Nourishment should be given freely, in a liquid form, and at frequent intervals; it should be always warm, cold drink being avoided for the same reason that cold air is excluded. Stimulants should be administered from the first in small quantities, say four or six ounces of brandy in the twenty-four hours, and increased if the pulse indicate it. This will hardly ever be necessary; for the pulse usually keeps up to the last, death, as I before stated, being rarely from exhaustion.

Third. Never give purgatives. It is obvious that when our object is to keep the nervous system quiet we should avoid purgatives of all kinds. Imagine the amount of irritation a dose of croton oil must cause; I have seen an exacerbation of all the symptoms occur after its exhibition. Fourth. Hydrate of chloral, together with extract of cannabis indica, is to be given in rapidly increasing doses, until the frequency and severity of the spasms are controlled. I generally commence with thirty grains of chloral in an ounce of water, and two grains of the extract of Indian hemp, in the form of pill, every three or four hours for an adult, and increase the former by fifteen grains and the latter by two grains until the desired effect is produced, when the spasms will be few and far between, the abdominal muscles almost normally flaccid, and the mouth opened to at least an inch ; the patient is then in a state of stupor from which he can be roused to take nourishment. I find that sixty orains of chloral and four grains of the extract is a full dose in fairly severe cases. It seems to me that the chloral controls the frequency of the attacks, and the hemp the intensity of the muscular contractions, and consequently the dose of either drug should be increased according to these indications. It is remarkable in some cases how the disease may be controlled in this manner. For instance, in Case 3 , on the sixteenth day, as there had been no spasms for seventy-two hours, I ordered the chloral to be discontinued. A few hours afterwards, in my presence, he had a severe spasm; the chloral was at once resumed, and there was no return of the disorder until the twenty-first day, when precisely the same thing again occurred. From this it will be seen how cautious we must be in leaving off the medicines. About the end of the second week, many cases are suddenly carried off by a spasm when they are thought to be out of danger. I am convinced that this happened in Case 1 , and that the nurse, imagining her patient safe, relaxed her watchfulness. From what I have seen I am inclined to lessen the frequency of the doses rather than their quantity when the patient gets over the second week, and this must be done with the utmost caution.

I do not claim for this treatment that it will be invariably successful. There are cases of tetanus, as there are cases of any other acute disease, that run their course to a fatal termination, apparently defying all attempts at treatment; but $I$ think the results of the above cases are encouraging, and hope on another occasion to record other successful case treated after the manner I have indicated.

While writing the above I have come across, in a pamphlet on tetanus, published in 1856, by Dr, Jackson, late Pre- sidency surgeon at Calcutta, the following passage, which singularly bears me out in the view I have taken of the treatment of tetanus. After stating that he had tried all methods of treatment without success, he goes on to say : "Ice to the spine, opium, camphor, I have tried, but I have no faith in them. I have found hemp and aloes much more beneficial, and from the use of chloroform I have seen patients derive great relief; but it was not until I had used this in combination with hemp and aloes, supporting the patient with good diet, quinine, and wine, and keeping him in a state of perfect rest, that I have met with anything like success."

I have already mentioned that death usually occurs in a spasm from apnœa. Now, even when the patient is apparently dead, we must not give up. Artificial respiration should be at once commenced and persevered with for a time; we should not wait even until the rigid muscles become partially relaxed, but the moment insensibility comes on an attempt may be made to get air into the lungs. I am convinced that many deaths from tetanus may thus be averted, at least for a time, and the patient given another chance of life.

\section{A CASE OF IDIOPATHIC TETANUS TREATED WITH ATROPIA AND CHLORAL HYDRATE ; RECOVERY.}

\section{BX E. WATSON PAUL, L.K.Q.C.P.}

THE following facts may be of sufficient interest to warrant their publication.

On Sept. 28th last, I was sent for to see a patient who "Could not open her mouth." Such was the message I received. On arrival $I$ found the patient (a young, unmarried woman) suffering from trismus, and spasm of the whole of the muscles of the neck, together with marked emprosthotonos. The patient's teeth were tightly clenched, and she could not speak, but was able to write a few words. There was no history of an injury; she referred her attack to having got her feet wet some ten days back. She had been ill five days before I was summoned. The catamenia were regular. I ordered her bowels to be immediately freed by an enema consisting of half an ounce of castor oil in a pint of warm water, after which ten grains of chloral hydrate were injected into the rectum, to be continued at intervals of an hour. Food (strong beef-tea and brandy in doses of from one to two ounces) was also administered by rectum.

Sept. 29th.-The"patient to-day is much worse; there is constant spasm of the muscles; emprosthotonos mich more marked; pulse weak and fluttering; countenance anxious, and symptoms altogether denoting failure of vital powers. I injected subcutaneously two minims of the solution of sulphate of atropia, made up to ten minims with glycerine and distilled water. Ordered nutrient enemata and chloral hydrate to be continued.

30th.-Patient still in a very weak state, but there was not so great exhaustion of the vital power as I was led to expect from the symptoms yesterday. The teeth are still tightly closed, and the rigidity of the muscles is very marked. The slightest sound brings on a violent paroxysm. I again injected the atropia as before.

Oct. 1st.-The patient seems a trifle better; the bowels were opened freely yesterday evening, and the pulse, if anything, is stronger. I injected the atropia as before, and ordered continuation of nutriment by the rectum.

2nd.-Slightly improved; the spasms are not so frequent, and the tongue can be protruded to the extent of an eighth of an inch between the teeth. The patient's friends, on their own responsibility, attempted to administer food by the mouth, and a violent paroxysm was the result. As the nutrient enemata have never been rejected, I ordered their continuance, also the chloral, and I again subcutaneously injected the atropia, this time increasing the dose to four minims of the solution.

3rd. - Patient's bowels not having been freed since Saturday, I ordered an enema as before. This had the desired effect. The tongue can still be protruded a short distance between the teeth, the pulse is firmer, and the paroxysms not so frequent. Attributing this improvement 
to the subcutaneous injections, I again resorted to them as on the previous day.

4th. -Patient still improving slowly but surely, but the spasms are still frequent, though not so severe. Treatment as before.

5th.-Decided improvement; patient can speak indistinctly; emprosthotonos subsiding; spasms not so frequent; pulse better. Did not inject the atropia to-day, but ordered combination of chloral hydrate.

6th.-Patient still improving. Decided to let her try a little food by the mouth, but without success. Injected five minims of solution of sulphate of atropia. Bowels naturally relieved to-day.

7th. - Patient improving slowly; spasms occasionally, but not so frequent or severe.

8th.-I again injected five minims of the atropia solution, and the patient expresses herself as feeling better. The tongue is very foul, and can be protruded about a quarter of an inch.

10th.-Bowels freely relieved naturally ; spasms fewer and less frequent; patient swallowed a little food by the mouth.

Ilth.-Improvement gradual, but sure. Discontinued the injections from this date, but continued the chloral hydrate. 12th.-No spasm for twenty-four hours ; tongue cleaning; food entirely administered by the mouth.

The patient got up for the first time on the 15th, and on the following day was convalescent.

Glastonbury, Somerset.

\section{KASE OF ACUTE TRAUMATIC TETANUS}

\section{By W. R. G. SAMUELS, M.D., F.A.S., M.R.C.S.}

If 1 As sent for on June 18th, at 12 P.M., to attend on H. H_, a youth seven years of age, of English parentage. He complained of stiffness in neek and jaws, with difliculty in swallowing. He had been apparently well up to this time. The tongue appeared somewhat swollen. I endeavoured to take a good look into the oral cavity, but was prevented from doing so, the patient being unable to open his mouth more than about an inch. The glands of the neck were somewhat enlarged and tense. He was supposed to have taken cold during the day, which would in part account for the above-named symptoms. I prescribed diaplooretics combined with purgatives; a gargle of chlorate of potash to be used frequently; hot poultices of linseed. meal to the throat, on which was sprinkled some turpentine, . 13 bottles of hot water to the feet. Crinated freely.

June 19tl. - 8 A.M.: The child appeared somewhat beter. I found him sitting at the window almost naked; rolled him in a blanket and placed him in bed. He was wiil unable to open his mouth any wider than on the night previons; urinated freely. At 1 P.M. I was sent for in haste, and found him much worse, with head thrown slightly bisk when the paroxysms came on, tonic contractions of the muscles of the neck, while the masseter muscles were haw and rigid. Ordered chloroform liniment to be applied to the throat and neck, with a mixture containing bromide of potash and chloral hydrate, twenty-grain doses to be given every hour. I kept the patient well ander the inhirence of the chloral hydrate, in order if possible to prevent the return of the spasms. The muscles of the abdomen ware normal. On my next visit, in company with another medical man, opisthotonos was found to be present to a wost alarming extent with each paroxysm. I had frequently inquired whether he had received any injury, and was rilways answered in the neoative. However, I was determined to find out, if possible, the truth of the statement, and on making a superficial examination of the body, 1 F und a spot on the outer and lower portion of the right leg

th ecchymotic state, with some hard substance embedded the muscle (peroneus). On making an incision, a splinter it red wood (pine) was discovered, which was found on exion to measture an inch and a quarter in length. From tuation I believe that it caused an irritation of the wi.isulo-cutaneous nerve, and, as a natural consequence, y refex action, produced tetanus, as already described. Imacle an incision in the direction the splinter had taken, ixiministered brandy freely, with hot poultices of linseednod to the wound, and continued the chloroform liniment as before, with chloral hydrate and bromide of potassium mixture. The mother for the first time now explained that the child had been complaining of a pain in his leg for about seven days; she had, however, taken but little notice of it, having been informed that a splinter had been removed some days previons. While I was present a paroxysm came on more violent than any of the preceding ones, arching his body nearly three-quarters of a circle in a posterior direction, and throwing him completely off the bed. After replacing him, I administered chloroform by inhalation. While under its influence the muscles of the throat and neck were relaxed, but on return to consciousness they were as rigid as before. During all this time he perspired freely, which I thought a somewhat favourable symptom.

20th. -8 A.M. : Muscular irritation and contraction not abated in the least. Slept several times during the night between each spasm, the interval being only a few minutes. Opisthotonos gradually getting worse, I made a $\mathbf{V}$-shaped incision a little above the seat of injury, passing the soalpel completely down to the bone, with a view of dividing the nerve. He was kept well under the influence of the chloral hydrate and bromide combined; and brandy was given every hour. Each movement in the room, touching or moving his body in the least, or the simple rustling of furniture, was immediately followed by a paroxysm. I subsequently used the galvanic battery, directing the current to the masseter muscles ; also applied the positive wire to the calf of the leg, while the negative was directed to the cervical spine. During the operation and almost immediately after breaking off the current the muscles were relaxed, but soon returned to their former condition. When the current was directed to the infra-orbital nerve, the mouth could be opened a little wider than before. It is scarcely needful for me to say that when the buccal muscles were operated on, distortion of the face was the resnlt. All this was of little or no avail, the muscles returning to their former abnormal condition. On my next visit, which was in the aftemoon, I was informed that during my absence the paroxysms were more frequent and violent until 4 P.M., when a spasm occurred more severe than the rest, and the boy had ceased to breathe; asphyxia doubtless being the cause of death. Had I been present when the last attack came on, in all probability I should have performed the operation of tracheotomy, in the hope of prolonging life.

This I consider to be a case of acute traumatic tetanus; the result of the splinter entering the leg setting up irritation. in the musculo-cutaneous nerve, and producing a reflex action on the spinal nerves, as quoted above. I was unable to try the effects of the Calabar bean. San Francisco.

\section{TETANUS TN A BOY, CAUSED BY THE STINGS OF THREE WASPS.}

\section{BY ARTHUR TUXFORD, M.D. EDIN.}

BENJAMIN R-, aged twelve years, son of a labourer, living at Boston West, was brought to me on Aug. 5th, 1877, suffering, as he said, from a stiff neck, with great difficulty of swallowing. His father told me that four days previously he had been stung by three wasps, receiving one sting under the lower jaw, another near the inner canthus of the right eye, and a third immediately above the right eye. No notice was taken of them until the evening of the 4th, when he complained of the symptoms stated. After examining him I ordered him to have a warm bath, to have the muscles of the neck and back rubbed with an ammonia liniment night and morning, to be followed by the application of a hot bread-and-water poultice; and, the bowels being confined, I administered a brisk purgative, which I after. wards found did not operate.

Next day I was fetched to the lad, who was stated to be as "stiff as a board, and unable to swallow anything." I found him as represented (in fact, he might have been held out by his heels), and with the back very much arched, his jaws firmly clenched, great difficulty of swallowing, hurried respiration, his body bathed in a cold clammy sweat, his legs and feet very cold, and his bowels still confined. I ordered ice-bags to his spine and bottles of hot water to the feet, bags of heated salt to the abdomen (which was very hard) and had a castor-oil injection administered. 